\title{
Smoking increases dental implant failures and complications
}

\author{
Abstracted from \\ Strietzel FP, Reichart PA, Kale A, Kulkarni M, Wegner B, Küchler I. \\ Smoking interferes with the prognosis of dental implant treatment: \\ a systematic review and meta-analysis. / Clin Periodontol 2007; 34: 523-544 \\ Address for correspondence: Frank Peter Strietzel, \\ Department of Oral Surgery and Dental Radiology, Campus Virchow Clinic, Centre for Dental Medicine, \\ Charité Medical University Berlin, Berlin, Germany. E-mail: frank.strietzel@charite.de
}

\section{Question: Does smoking affect the prognosis of dental implants?}

\begin{abstract}
Data Sources Medline, Pubmed, Embase and the references of retrieved articles were used to identify relevant data. Study selection Clinical studies were included for meta-analysis if they compared implant or patient-related data on failures of implant treatment in relation to the numbers of smokers and nonsmokers. Published clinical studies providing statistically examined data [odds ratios (OR), risk ratios or hazard ratios] of implant failures or biological complications among smokers compared with nonsmokers were included for systemic analysis.

Data extraction and synthesis Screening of eligible studies was carried out by two independent reviewers. Patient-related studies and implantrelated studies were analysed separately. Heterogeneity was investigated and publication bias assessed using funnel plots. Additional data extracted, when available, included the sex of the patient, mandibular or maxillary placement, and any augmentation measures. These dichotomous outcomes were expressed as risk ratios with $95 \%$ confidence intervals (Cl). Post implant observation periods were categorised for analysis. Results In all, 29 studies were included for meta-analysis and 35 for systematic review. Meta-analysis revealed a significantly enhanced risk for implant failure, with or without augmentation, in smokers. The systemic review showed significantly enhanced risks of peri-implant complications and bone loss in smokers. In five studies, in which implants had particle-blasted, acid-etched or anodic oxidised surfaces, smoking had no significant impact on the prognosis of such implants. Conclusions The risk of implant failures and biological complications with and without accompanying augmentation procedures was found to be significantly increased in smokers compared with nonsmokers.
\end{abstract}

\section{Commentary}

Systematic reviews start with a narrow, focused question which dictates the types of studies that will meet the inclusion criteria. The authors here searched for trials, cohorts and case-control studies, an approach not supported by the nature of the clinical question. The clinical question in this review asks if there is a difference in implant success rates in smokers versus nonsmokers. This is a not a question of which therapy is better; rather, it is a question of prognosis (cohort studies) or risk factor/ exposure (case-control studies), and systematic reviews of observa- tional studies present many challenges compared with reviews of randomised trials.

The process of randomisation in trials essentially eliminates all confounders found in observational studies, especially cohort studies. For example, if one looked for an association between periodontitis and cancer, it is likely to occur, as both are associated with ageing. It would be difficult to conclude, however, that one caused the other. Ageing, then, is a confounder, and although it is common to show an association between two variables, a number of criteria need to be met to show causality. One of these criteria is biological plausibility, as smoking has been identified as a major risk factor for the development of periodontitis.

Bias can also be an issue in trials, but bias in case-control designs can easily lead to misleading results. The inclusion of case-control studies in systematic reviews can lead to bias because of difficulties in recall of smoking habits as well as the selection of control subjects.

In this study, smokers were not classified according to the numbers of cigarettes smoked.

There was no mention of the use of data extraction sheets, which can be helpful for critical appraisal of studies considered for inclusion. One of the most important tasks in a systematic review is to assess for bias each of the studies included. This can be done with a scoring system or a qualitative description of study bias. There was no mention here of assessment of study bias. Although a Cohen's Kappa statistic was provided for inter-examiner agreement, it is difficult to know how each included study was assessed for quality. This is a critical omission, as the inclusion of many potentially low-quality studies will make for a low-quality review. Additionally, assessing the quality of observational designs is more difficult than for trials. ${ }^{1}$

It is generally contraindicated to combine results of observational studies into a meta-analysis as the results may be misleading because of both confounders and bias. Combining studies statistically (meta-analysis) is inappropriate if extreme heterogeneity exists. Assessment of studies for heterogeneity is critical, as combining studies with clinical and methodological differences can lead to spurious results. The characteristics of studies did not mention the specific study design, and a subgroup analysis, which separated cohort from case-control, would have been useful to examine differences in OR between these two designs. In this case, heterogeneity was measured and found to be present, which necessitated the use of a random effects model. Individual patient data may have been useful in providing a summary estimate of effects in this review, in lieu of a meta-analysis. 


\section{PERIODONTAL DISEASE}

It appeared that included studies were limited to those reported in English or German, and there was no mention of searches for unpublished studies, using bibliographies of included studies or contacting experts for additional articles. This is relevant, as the potential for omitting unpublished studies particularly risks biasing the results in favour of significance as, often, studies with nonsignificant results are not submitted for publication. The authors used a funnel plot to examine publication bias, which showed symmetry around the null value, however, indicating studies with nonsignificant results were not omitted from the review.

A subgroup analysis was performed for both observation period and site augmentation. To their credit, the authors separated failures into both implant-related and patient-related failures.

The overall results from this study were as follows:

- Overall implant-related OR of implant failure was 2.25 (95\% CI, 1.96-2.59).

- Overall patient-related OR of 2.64 (95\% CI, 1.70-4.09).

- Random effects model of implant failure because of heterogeneity among studies was 2.38 (95\% CI, 1.93-2.93) which typically shows a wider CI, as studies are measuring different effects.

- OR for implant failure in smokers for an observation period of up to 1 year was 2.83 (95\% CI, 2.08-3.85).

- OR for implant failure in smokers for an observation period of more than 5 years was 1.33 (95\% CI, 0.84-2.11) which is not statistically significant, indicating that there is greater risk of early implant failure in smokers.

- A random effects model of site augmentation procedures gave an OR of 2.15 (95\% CI, 1.86-2.49) for non-augmented sites and an OR of 3.61 (95\% CI, 2.26-5.77) for augmented sites, showing higher risk of implant failure in smokers where site augmentation was performed.

The interpretation of an OR of 2.25 means that the odds of implant failure in a smoker are more than twice (2.25-fold) the odds in a nonsmoker. This is statistically significant as the CI does not include the null value of 1 . The clinical relevance requires further consideration, however. If overall implant success rates are approaching 95\% ${ }^{3-5}$ then success rates in smokers may be around 90\% as OR are similar to risk ratios when outcomes (failure) are relatively uncommon. Thus, information given to smokers should include increased risk of failure compared with nonsmokers, yet with close followup, especially in the first year post-operatively, failure may be infrequent.

Results from this review are remarkably similar to a good-quality systematic review by Hinode et al. ${ }^{2}$ This review used a subgroup analysis to examine success in the maxilla versus the mandible. Whereas the overall OR for implant failure was 2.17 (95\% CI, 1.67-2.83), the OR in the maxilla was 2.06 (95\% CI, 1.61-2.65) and in the mandible was 1.32 (95\% CI, 0.72-2.40), meaning the odds of failure was double in the maxilla, but statistically insignificant in the mandible.

These two systematic reviews represent a small but growing body of evidence indicating implant failure risk is higher in smokers than in nonsmokers. As the risk of implant failure is generally low, individual practitioners will have to decide what modifications to therapy, if any, should be employed with their patients.

Elliot Abt

Department of Dentistry, Illinois Masonic Medical Center, Chicago, Illinois, USA

1. Altman D. Systematic reviews in Health Care: Meta-analysis in Context. Chapter 13. Systematic Reviews of Prognostic Variables. Edited by M Egger, G Davey Smith, DG Altman. London: BMJ Books; 2003.

2. Hinode D Tanabe S, Yokoyama M, Fujisawa K, Yamauchi E, Miyamoto Y. Influence of smoking on osseointegrated implant failure: a meta-analysis. Clin Oral Impl Res 2006; 17: $473-478$.

3. Lindh T, Gunne J, Tillberg A, Molin M. A meta-analysis of implants in partial edentulism. Clin Oral Impl Res 1998; 9: 80-90.

4. Attard NJ, Zarb G. Implant prosthodontic management of partially edentulous patients missing posterior teeth: the Toronto experience. J Prosth Dent 2003; 89: 352-359.

5. Creugers NH, Kreulen CM, Snoek PA, de Kanter RJ. A Systematic review of single-tooth restorations supported by implants. J Dent 2000; 28: 209-217.

Evidence-Based Dentistry (2009) 10, 79-80. doi:10.1038/sj.ebd.6400670 